\title{
Conducting Randomized Trials in the Electrophysiology Laboratory: Lessons from a Randomized Comparison of Recording Methods During Pulmonary Vein Isolation by Segmental Ostial Ablation
}

\author{
Hakan Oral and Fred Morady \\ Division of Cardiology, University of Michigan, \\ Ann Arbor, MI, USA
}

\begin{abstract}
Accurate identification of pulmonary vein $(P V)$ potentials during segmental ostial ablation for PV isolation is very important to completely isolate the PVs and also to avoid unnecessary applications of radiofrequency energy. A prior post hoc analysis of unipolar and bipolar electrograms recorded from successful and unsuccessful ablation sites demonstrated that unipolar electrograms recorded at successful sites were more likely to have a rapid intrinsic deflection, larger amplitude, and earlier activation than electrograms recorded at unsuccessful sites. Moreover, unipolar electrograms recorded from the ablation and circular ostial mapping catheters were almost identical at successful sites. Based on these observations, a prospective, randomized study was conducted to test whether unipolar and bipolar electrograms would facilitate the ablation procedure when compared to bipolar electrograms alone during $P V$ isolation in patients with atrial fibrillation (AF). In 44 consecutive patients with paroxysmal AF, 114 PVs were randomized to segmental ostial ablation guided by unipolar and bipolar electrograms (61) or by bipolar electrograms only (53). Segmental ostial ablation guided by unipolar and bipolar electrograms was associated with $a \sim 20-30 \%$ decrease in the procedure and fluoroscopy times necessary for isolation of a PV and also in the duration of radiofrequency energy application required for complete isolation of a PV. Although the sample size was not sufficient to detect a $5 \%$ change, the success rate for complete electrical isolation and the risk of symptomatic PV stenosis were similar between the 2 groups. Online analysis of unipolar electrograms facilitated the PV isolation procedure and was incremental to the analysis of bipolar electrograms alone. However, because segmental ostial ablation has only modest efficacy in achieving long-term freedom from recurrent AF, alternative ablation strategies that may or may not target PVs will eventually evolve. The role of unipolar electrograms in these new methods remains to be determined.
\end{abstract}

Key Words. atrial fibrillation, pulmonary vein, catheter ablation, electrogram, unipolar

After the observation that arrhythmogenic activity within the pulmonary veins (PV) may play a critical role in the initiation [1] and perpetuation of atrial fibrillation (AF) $[2,3]$, the PVs have been targeted in patients with paroxysmal or persistent AF. After the initial attempts of focal ablation with applications of radiofrequency energy within a PV resulted in a high recurrence and PV stenosis rate, several ablation strategies have been proposed to isolate the pulmonary veins [4-8]. One of the first and most widely used methods was segmental ostial ablation by applications of radiofrequency energy at ostial sites where there is an electrical connection between the left atrium and the pulmonary vein $[3,4,9]$.

\section{Pulmonary Vein Isolation by Segmental Ostial Ablation}

During segmental ostial ablation, accurate identification of pulmonary vein potentials is of critical importance. Around the ostium of a pulmonary vein, double potentials usually are recorded, the first component being due to left atrial depolarization and the second component being due to activation of a pulmonary vein fascicle. These potentials often are fused. Although several maneuvers such as pacing from the coronary sinus or the left atrial appendage, or introducing a premature stimulus may facilitate identification of PV potentials [10-12], these maneuvers at times are time-consuming and may not always be helpful.

Failure to accurately identify pulmonary vein potentials may result in incomplete isolation of the PVs, unnecessary and excessive applications of radiofrequency energy, and prolongation of the procedure. The accurate identification of PV potentials is critical for the success and safety of the ablation procedure.

Because unipolar recordings precisely reflect local activation, it is possible that the analysis of

Supported by the Ellen and Robert Thompson Atrial Fibrillation Research Fund.

Address correspondence to: Hakan Oral, M.D., Cardiology, TC B1 140D, 1500 E. Medical Center Dr., Ann Arbor, MI 481090311, USA. E-mail: oralh@umich.edu 
unipolar electrograms in addition to bipolar electrograms recorded at the PV ostia could facilitate the accurate identification of the PV potentials.

\section{Background: Post hoc analysis of unipolar and bipolar electrograms during segmental ostial ablation for PV isolation}

As a first step in determining whether unipolar recordings have any value during a PV isolation procedure, unipolar and bipolar electrograms recorded at successful and unsuccessful PV ostial ablation sites were compared. A successful site was defined as an ostial site where an application of radiofrequency energy resulted in elimination of the targeted $\mathrm{PV}$ potential(s). In 21 patients who underwent a PV isolation procedure for AF, unipolar and bipolar electrograms recorded with a decapolar circular catheter positioned at the ostium of the right superior, left superior, and left inferior PV and with a quadripolar ablation catheter with a 4-mm-tip electrode positioned at the ostial side of the decapolar catheter were analyzed retrospectively. Bipolar electrograms were recorded at a bandpass of $30-500 \mathrm{~Hz}$, whereas unipolar electrograms were recorded at $0.5-200 \mathrm{~Hz}$. The intervals between the onset of the $P$ wave or the pacing stimulus and the local electrogram, the interval between the atrial electrogram and PV potentials, the morphologic characteristics of the electrograms, the duration and amplitude of the electrograms and the slope of the intrinsic deflection of the unipolar electrogram were measured using digital calipers (Figure 1) [13]. PV potentials were defined as depolarizations with a rapid upstroke, a duration

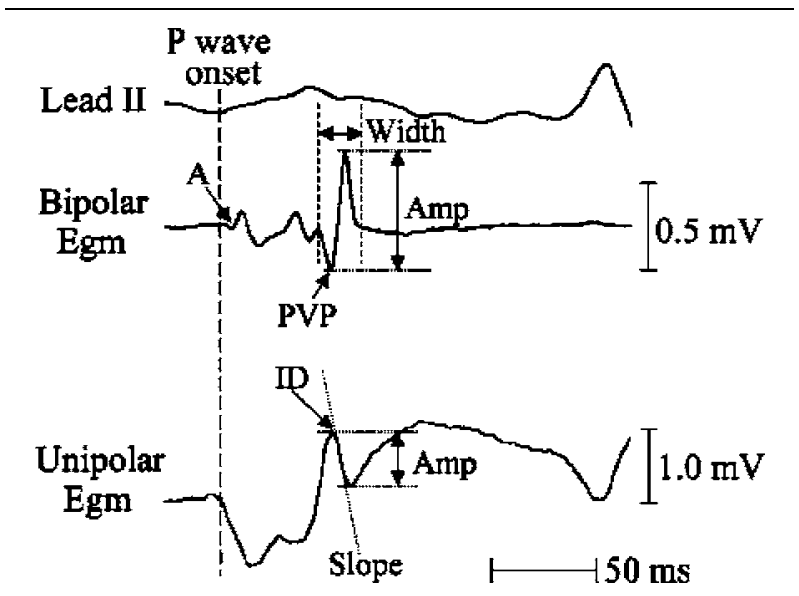

Fig. 1. Bipolar and unipolar electrograms of pulmonary vein potentials. Bipolar and unipolar electrograms recorded from a circular decapolar catheter are shown. The electrograms were analyzed using digital calipers. Reproduced with permission from reference 13. Abbreviations: Amp = amplitude, Egm = electrogram, $A=$ atrial electrogram, Amp = amplitude, $P V P=$ pulmonary vein potential, $I D=$ intrinsic deflection.
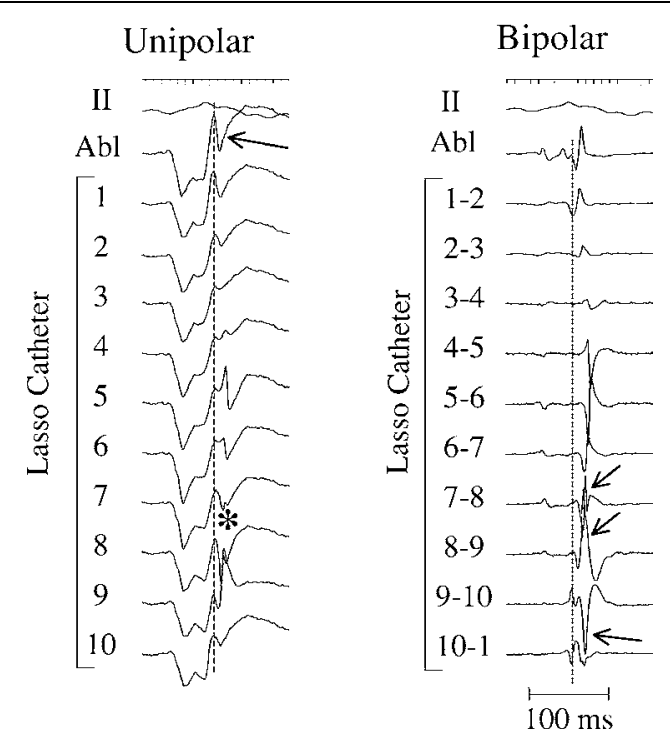

Fig. 2. Identification of ablation sites with the guidance of bipolar and unipolar/bipolar electrograms. Bipolar and unipolar electrograms recorded from a decapolar circular catheter positioned within the ostium of a PV are shown. Bipolar electrogram recorded from the ablation catheter precedes the electrograms recorded from the decapolar catheter (Panel A). In Panel B, unipolar electrogram recorded from the ablation catheter is earlier that the unipolar electrogram with the steepest intrinsic deflection recorded from electrode 4 of the decapolar catheter. In addition the unipolar electrograms recorded from the ablation catheter and electrode 4 are similar suggesting that ablation catheter is very close to the target site. Reproduced with permission from reference 14.

$<50 \mathrm{~ms}$, and an amplitude $>0.05 \mathrm{mV}$ that followed a left atrial depolarization.

Radiofrequency energy was applied at ostial sites where a PV potential earlier than the earliest potential recorded with the decapolar ring catheter was identified with the ablation catheter (Figure 2) [13]. Electrograms recorded from 185 successful and 120 unsuccessful sites were analyzed and compared.

When bipolar electrograms recorded from the successful and unsuccessful sites were compared, the PV potentials recorded at the successful sites were earlier and had a larger amplitude (Figures 2 and 3) [13]. Likewise when unipolar electrograms recorded at successful and unsuccessful sites were compared, the unipolar electrograms recorded at the successful sites were earlier and had a larger amplitude. Moreover, the intrinsic deflection of the unipolar electrogram was steeper at successful sites. Another observation was that the unipolar electrograms recorded by the ablation catheter were more likely to be identical to the electrogram recorded from the targeted electrode of the decapolar catheter at successful ostial sites than at unsuccessful sites (Figure 3). Among all variables, a rapid intrinsic deflection of the 


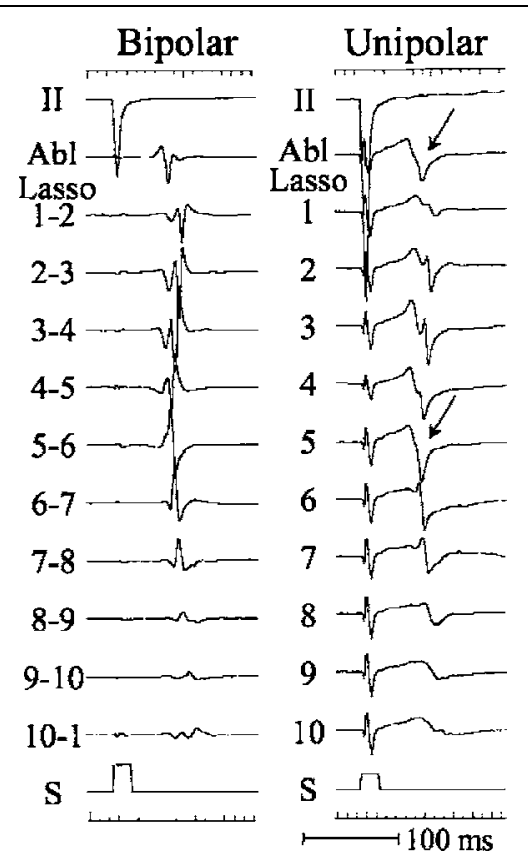

Fig. 3. Identification of the site of earliest activation with unipolar electrograms. Bipolar and unipolar electrograms recorded from a circular decapolar catheter positioned within $5 \mathrm{~mm}$ of the ostium of the left superior pulmonary vein are shown. Analysis of unipolar electrograms shows that the earliest activation occurs at electrode 5 as manifest by the rapid intrinsic deflection preceding all other electrograms. It is important to note that the morphology of the unipolar electrograms recorded from the ablation catheter and electrode 5 are similar suggesting that the ablation catheter is in close proximity of the target site. Reproduced with permission from reference 13 .

unipolar electrogram $(\leq-0.08 \mathrm{mV} / \mathrm{ms})$ had the best sensitivity and specificity for identifying a successful ablation site (71 and 90\%, respectively). Although a bipolar or unipolar electrogram amplitude $\geq 1 \mathrm{mV}$ had a specificity of $\leq 90 \%$, the sensitivity was only $\sim 25 \%$.

The findings of this study suggested that unipolar electrograms with rapid intrinsic deflections had a high diagnostic accuracy for identifying successful ostial sites. In addition, the findings suggested that a morphology match between the unipolar electrograms recorded by the ablation catheter and the ring catheter might be useful in accurately positioning the ablation catheter.

\section{Hypothesis}

Based on these findings of the post hoc analysis, we hypothesized that the online analysis of unipolar electrograms during segmental ostial ablation to isolate the pulmonary veins would facilitate the accurate identification of PV potentials.

\section{Study Design: A Prospective and Randomized Clinical Study}

To test this hypothesis, a prospective, randomized study was designed. During ablation procedures for paroxysmal AF, the left superior, left inferior and right superior PVs were randomly assigned to segmental ostial ablation guided by unipolar and bipolar electrograms, or only bipolar electrograms. To eliminate the confounding variable of differences in the anatomy and electrophysiologic substrate between the study subjects, individual PVs were randomized rather than the patients. Because unipolar electrograms are not feasible for very low amplitude local potentials [13], and because the purpose of the unipolar electrograms was to facilitate the identification of PV potentials rather than to replace bipolar electrograms, analysis of bipolar electrograms was allowed in the unipolar arm of the study. The right inferior pulmonary vein was not included in this study because it could not be consistently cannulated with the ring catheter.

Segmental ostial ablation was performed during sinus rhythm or pacing from the coronary sinus, as described previously $[9,14]$. The study endpoints consisted of: (1) complete electrical isolation; (2) duration of time necessary for isolation of an individual PV; (3) fluoroscopy time per PV; (4) duration of radiofrequency energy required to achieve complete electrical isolation; and (5) PV stenosis.

A sample size analysis revealed that $50 \mathrm{PVs}$ in each group would be required to detect a $20 \%$ change in the procedural parameters of duration of radiofrequency energy applications, amount of fluoroscopy, and procedure times with a power of $80 \%$ and an $\alpha$ of 0.05 . At the same settings, $>400 \mathrm{PVs}$ would be necessary to detect a $5 \%$ change in complete electrical isolation of the PVs and the risk of PV stenosis.

\section{Segmental Ostial Ablation Guided by Unipolar and/or Bipolar Electrograms: Main Findings}

Complete electrical isolation was achieved in $96 \%$ of the $53 \mathrm{PVs}$ randomized to the bipolar electrogram approach and in $93 \%$ of the $61 \mathrm{PVs}$ randomized to the unipolar-bipolar electrogram approach $(p=0.7)$. The duration of the ablation procedure and the total amount of fluoroscopy were 19 and $28 \%$ shorter when ablation was guided by the analysis of unipolar and bipolar electrograms than when guided by only bipolar electrograms [14]. Moreover, the total amount of radiofrequency energy required to achieve complete electrical isolation was $28 \%$ shorter with the unipolar-bipolar approach than with the bipolar approach alone [14].

Maximum power was limited to 35 watts and the temperature was limited to $52^{\circ} \mathrm{C}$ during ostial 
ablation of PVs. There were no instances of PV stenosis regardless of whether ablation was guided by unipolar/bipolar or bipolar electrograms.

\section{Clinical Implications}

The findings of this prospective, randomized study suggest that when unipolar electrograms are analyzed in conjunction with bipolar electrograms, the procedure time, amount of fluoroscopy, and amount of radiofrequency energy needed to completely isolate the PVs by segmental ostial ablation are significantly reduced. More accurate identification of PV potentials is possible because unipolar recordings eliminate the influence of far-field potentials. This may be particularly important in PVs because of the complex, multi-layer, 3-dimensional architecture of the muscle sleeves that surround the pulmonary veins [15]. Another advantage of the unipolar recordings is that the ostial location of the ablation catheter relative to the electrodes of the ring catheter can be determined by comparing the morphologies of the electrograms recorded by the ablation and ring catheters $[13,14]$.

\section{Future Directions}

These 2 studies demonstrated the incremental value of unipolar electrograms to bipolar electrograms during segmental ostial ablation for PV isolation. However, segmental ostial ablation in general has been reported to have an efficacy of $\sim 70 \%$ for long-term freedom from recurrent $\mathrm{AF}[3,4,9,16]$, and repeat ablation procedures may be necessary in up to $40 \%$ of patients with paroxysmal AF. Furthermore, segmental ostial ablation has poor efficacy in patients with persistent AF. Therefore, although segmental ostial ablation may continue to play an important role in the treatment of atrial fibrillation, it is likely that it will need to be supplemented by additional ablation directed at the left atrial substrate [17]. Left atrial ablation to encircle the pulmonary veins also has been shown to be effective in eliminating atrial fibrillation, and this type of anatomic approach to ablation precludes the need for electrogram analysis [6].

\section{References}

1. Haissaguerre M, Jais P, Shah DC, Takahashi A, Hocini M, Quiniou G, Garrigue S, Le Mouroux A, Le Metayer P, Clementy J. Spontaneous initiation of atrial fibrillation by ectopic beats originating in the pulmonary veins. $N$ Engl J Med 1998;339:659-666.

2. Oral H, Ozaydin M, Tada H, Chugh A, Scharf C, Hassan S, Lai S, Greenstein R, Pelosi F Jr, Knight BP, Strickberger SA, Morady F. Mechanistic significance of intermittent pulmonary vein tachycardia in patients with atrial fibrillation. J Cardiovasc Electrophysiol 2002;13:645-650.
3. Oral H, Knight BP, Ozaydin M, Chugh A, Lai SW, Scharf C, Hassan S, Greenstein R, Han JD, Pelosi F Jr, Strickberger SA, Morady F. Segmental ostial ablation to isolate the pulmonary veins during atrial fibrillation: Feasibility and mechanistic insights. Circulation 2002;106:1256-1262.

4. Haissaguerre M, Shah DC, Jais P, Hocini M, Yamane T, Deisenhofer I, Chauvin M, Garrigue S, Clementy J. Electrophysiological breakthroughs from the left atrium to the pulmonary veins. Circulation 2000;102:2463-2465.

5. Pappone C, Rosanio S, Oreto G, Tocchi M, Gugliotta F, Vicedomini G, Salvati A, Dicandia C, Mazzone P, Santinelli V, Gulletta S, Chierchia S. Circumferential radiofrequency ablation of pulmonary vein ostia: A new anatomic approach for curing atrial fibrillation. Circulation 2000;102:2619_ 2628.

6. Pappone C, Oreto G, Rosanio S, Vicedomini G, Tocchi M, Gugliotta F, Salvati A, Dicandia C, Calabro MP, Mazzone P, Ficarra E, Di Gioia C, Gulletta S, Nardi S, Santinelli V, Benussi S, Alfieri O. Atrial electroanatomic remodeling after circumferential radiofrequency pulmonary vein ablation: Efficacy of an anatomic approach in a large cohort of patients with atrial fibrillation. Circulation 2001;104:2539-2544.

7. Natale A, Pisano E, Shewchik J, Bash D, Fanelli R, Potenza D, Santarelli P, Schweikert R, White R, Saliba W, Kanagaratnam L, Tchou P, Lesh M. First human experience with pulmonary vein isolation using a throughthe-balloon circumferential ultrasound ablation system for recurrent atrial fibrillation. Circulation 2000;102:18791882.

8. Mangrum JM, Mounsey JP, Kok LC, DiMarco JP, Haines DE. Intracardiac echocardiography-guided, anatomically based radiofrequency ablation of focal atrial fibrillation originating from pulmonary veins. $J$ Am Coll Cardiol 2002;39:1964-1972.

9. Oral H, Knight BP, Tada H, Ozaydin M, Chugh A, Hassan S, Scharf C, Lai SW, Greenstein R, Pelosi F Jr, Strickberger SA, Morady F. Pulmonary vein isolation for paroxysmal and persistent atrial fibrillation. Circulation 2002;105:1077-1081.

10. Tada H, Oral H, Greenstein R, Pelosi F Jr, Knight BP, Strickberger SA, Morady F. Differentiation of atrial and pulmonary vein potentials recorded circumferentially within pulmonary veins. J Cardiovasc Electrophysiol 2002;13:118-123.

11. Tada H, Oral H, Ozaydin M, Greenstein R, Pelosi F Jr, Knight BP, Strickberger SA, Morady F. Response of pulmonary vein potentials to premature stimulation. $J$ Cardiovasc Electrophysiol 2002;13:33-37.

12. Shah D, Haissaguerre M, Jais P, Hocini M, Yamane T, Macle L, Choi KJ, Clementy J. Left atrial appendage activity masquerading as pulmonary vein potentials. Circulation 2002;105:2821-2825.

13. Tada H, Oral H, Wasmer K, Greenstein R, Pelosi F Jr, Knight BP, Strickberger SA, Morady F. Pulmonary vein isolation: Comparison of bipolar and unipolar electrograms at successful and unsuccessful ostial ablation sites. J Cardiovasc Electrophysiol 2002;13:13-19.

14. Tada H, Oral H, Knight BP, Ozaydin M, Chugh A, Scharf C, Hassan S, Greenstein R, Pelosi F Jr, Strickberger SA, Morady F. Randomized comparison of bipolar versus unipolar plus bipolar recordings during segmental ostial ablation of pulmonary veins. J Cardiovasc Electrophysiol 2002;13:851-856. 
15. Saito T, Waki K, Becker AE. Left atrial myocardial extension onto pulmonary veins in humans: Anatomic observations relevant for atrial arrhythmias. J Cardiovasc Electrophysiol 2000;11:888-894.

16. Macle L, Jais P, Weerasooriya R, Hocini M, Shah DC, Choi KJ, Scavee C, Raybaud F, Clementy J, Haissaguerre M. Irrigated-tip catheter ablation of pulmonary veins for treatment of atrial fibrillation. J Cardiovasc Electrophys- iol 2002;13:1067-1073.

17. Jais P, Hocini M, Sanders P, Hsu LF, Scavee C, Weerasooriya R, Sacher F, Reuter S, Garrigue S, Clementy J, Haissaguerre M. Importance of epicardial ablation through the coronary sinus to complete mitral isthmus block in ablation of paroxysmal atrial fibrillation. Pacing Clin Electrophysiol 2003;26: Abstract. 